\title{
Existence and uniqueness of solutions for nonlinear impulsive differential equations with three-point boundary conditions
}

\author{
Misır J. Mardanov, Yagub A. Sharifov and Kamala E. Ismayilova \\ Communicated by Allaberen Ashyralyev
}

\begin{abstract}
This paper is devoted to a system of nonlinear impulsive differential equations with three-point boundary conditions. The Green function is constructed and considered original problem is reduced to the equivalent impulsive integral equations. Sufficient conditions are found for the existence and uniqueness of solutions for the boundary value problems for the first order nonlinear system of the impulsive ordinary differential equations with three-point boundary conditions. The Banach fixed point theorem is used to prove the existence and uniqueness of a solution of the problem and Schaefer's fixed point theorem is used to prove the existence of a solution of the problem under consideration. We illustrate the application of the main results by two examples.
\end{abstract}

Keywords. Three-point boundary conditions, existence, uniqueness, fixed point, impulsive differential equations.

2010 Mathematics Subject Classification. 34B10, 34B37, 34A37.

\section{Introduction}

The theory of impulsive differential equations is a relatively new and crucial branch of differential equations. The theory has found applications in many areas where evolutionary processes undergo rapid changes at certain times of their development. There has been a great deal of development in impulsive theory, especially in the area of impulsive differential equations with fixed point moments (see, for instance, the monographs [1-4] and the references therein). We must note here that there are applications of impulsive differential equations to intervention models and interrupted time series analysis, percussive systems with vibrations to population dynamics, and relaxation oscillations of the electromechanical systems ([6-8] and the references therein). Some authors have produced an extensive portfolio of results on differential equations undergoing impulse effects. The existence questions for impulsive differential equations have been studied in [10-19] and references therein. Three-point boundary value problems for ordinary differential 
equations are also studied in recent years. They arise in the modeling and analyzing of many physical systems, such as some problems in the theory of elastic stability, the vibrations of a guy-wire of a uniform cross-section and composed on $N$ parts of different densities [5]. Moreover, boundary value problems have been studied by a number of authors in [9,20-29] and references therein.

\section{Problem statement}

This paper deals with the existence and uniqueness of solution for the system of nonlinear impulsive differential equations of the type

$$
\dot{x}(t)=f(t, x(t)) \text { for } t \neq t_{i}, i=1,2, \ldots, p, t \in[0, T],
$$

subject to impulsive conditions

$$
\begin{gathered}
x\left(t_{i}^{+}\right)-x\left(t_{i}\right)=I_{i}\left(x\left(t_{i}\right)\right), i=1,2, \ldots, p, t \in[0, T], \\
0=t_{0}<t_{1}<\ldots<t_{p_{1}}<\tau<t_{p_{1+1}}<\ldots<t_{p}<t_{p+1}=T,
\end{gathered}
$$

and three-point boundary conditions

$$
A x(0)+B x(\tau)+C x(T)=d,
$$

where $A, B, C$ are constant square matrices of order $n$ such that $\operatorname{det} N \neq 0, N=$ $(A+B+C) ; f:[0, T] \times R^{n} \rightarrow R^{n}$ and $I_{i}: R^{n} \rightarrow R^{n}$ are given functions; $\Delta x\left(t_{i}\right)=x\left(t_{i}^{+}\right)-x\left(t_{i}^{-}\right)$, where

$$
x\left(t_{i}^{+}\right)=\lim _{h \rightarrow 0+} x\left(t_{i}+h\right), x\left(t_{i}^{-}\right)=\lim _{h \rightarrow 0+} x\left(t_{i}-h\right)=x\left(t_{i}\right)
$$

are the right- and left-hand limits of $x(t)$ at $t=t_{i}$, respectively.

In the following, we first introduce necessary background. Next, theorems related to existence and uniqueness of a solution of problem (1)-(3) are proved under some sufficient conditions on the nonlinear terms. At last, an example of application of the main result of this paper is given.

\section{Preliminaries}

In this section, we give notations, preliminary facts and basic definitions which are used throughout this paper. We denote by $C\left([0, T] ; R^{n}\right)$, the Banach space of all vector continuous functions $x(t)$ from $[0, T]$ into $R^{n}$ with the norm

$$
\|x\|=\max \{|x(t)|: t \in[0, T]\},
$$


where $|\cdot|$ is the norm in the space $R^{n}$.

We consider the linear space

$$
P C\left([0, T], R^{n}\right)=\left\{x:[0, T] \rightarrow R^{n}: x(t) \in C\left(\left(t_{i}, t_{i+1}\right], R^{n}\right), i=0,1, \ldots, p,\right.
$$

$x\left(t_{i}^{-}\right)$and $x\left(t_{i}^{+}\right)$exist $i=1, \ldots, p$ and $\left.x\left(t_{i}^{-}\right)=x\left(t_{i}\right)\right\}$.

It is clear that $P C\left([0, T], R^{n}\right)$ is a Banach space with the norm

$$
\|x\|_{P C}=\max \left\{\|x\|_{\left(t_{i}, t_{i+1}\right]}, i=0,1, \ldots, p\right\} .
$$

Now we study the problem (1)-(3). We give first the definition of solution of the problem(1)-(3).

Definition 3.1. A function $x \in P C\left([0, T], R^{n}\right)$ is said to be a solution of problem (1)-(3) if $\dot{x}(t)=f(t, x(t))$ for each $t \neq t_{i}, i=1,2, \ldots, p, t \in[0, T] ; x\left(t_{i}^{+}\right)-$ $x\left(t_{i}\right)=I_{i}\left(x\left(t_{i}\right)\right)$ for each $i=1,2, \ldots, p, t \in[0, T], 0=t_{0}<t_{1}<\ldots<t_{p_{1}}<$ $\tau<t_{p_{1+1}}<\ldots<t_{p}<t_{p+1}=T$, and boundary conditions (3) are satisfied.

Lemma 3.2. Let $y \in C\left([0, T], R^{n}\right)$ and $a_{i} \in R^{n}, i=1,2, \ldots, p$, then the unique solution $x(t) \in C\left([0, T], R^{n}\right)$ of the boundary value problem for impulsive differential equation

$$
\begin{gathered}
\dot{x}(t)=y(t), t \in[0, T], t \neq t_{i}, i=1,2, \ldots, p, \\
x\left(t_{i}^{+}\right)-x\left(t_{i}\right)=a_{i}, i=1,2, \ldots, p \\
0<t_{1}<\ldots<t_{p_{1}}<\tau<t_{p_{1+1}}<\ldots<t_{p}<t_{p+1}=T, \\
A x(0)+B x(\tau)+C x(T)=d,
\end{gathered}
$$

is given by

$$
x(t)=D+\int_{0}^{T} G(t, s) y(s) d s+\sum_{0<t_{k}<T} G\left(t_{i}, t_{k}\right) a_{k}
$$

for $t \in\left(t_{i}, t_{i+1}\right], i=0,1, \ldots, p, D=N^{-1} d$, where

$$
G(t, s)=\left\{\begin{array}{lc}
G_{1}(t, s), & 0 \leq t<\tau \\
G_{2}(t, s), & \tau \leq t \leq T
\end{array}\right.
$$

with

$$
G_{1}(t, s)=\left\{\begin{array}{lr}
N^{-1} A, & 0 \leq s \leq t, \\
-N^{-1}(B+C), & t \leq s \leq \tau, \\
-N^{-1} C, & \tau<s \leq T
\end{array}\right.
$$


and

$$
G_{2}(t, s)=\left\{\begin{array}{lr}
N^{-1} A, & 0 \leq s \leq \tau \\
N^{-1}(A+B), & \tau<s \leq t \\
-N^{-1} C, & t<s \leq T
\end{array}\right.
$$

Proof. Assume that $x(t)$ is a solution of boundary value problem (4)-(6). Then integrating equation (4) for $t \in\left(0, t_{j+1}\right)$, we obtain

$$
\begin{aligned}
\int_{0}^{t} y(s) d s & =\int_{0}^{t} x^{\prime}(s) d s=\left[x\left(t_{1}\right)-x\left(0^{+}\right)\right]+\left[x\left(t_{2}\right)-x\left(t_{1}^{+}\right)\right]+\ldots \\
& +\left[x(t)-x\left(t_{j}^{+}\right)\right]=-x(0)-\left[x\left(t_{1}^{+}\right)-x\left(t_{1}\right)\right] \\
- & {\left[x\left(t_{2}\right)^{+}-x\left(t_{2}\right)\right]-\ldots-\left[x\left(t_{j}^{+}\right)-x\left(t_{j}\right)\right]+x(t) . }
\end{aligned}
$$

Using the above formula and condition (5), we can write

$$
x(t)=x(0)+\int_{0}^{t} y(s) d s+\sum_{0<t_{j}<t} a_{j} .
$$

In order for (7) to satisfy the condition (6), we get

$$
\begin{aligned}
& (A+B+C) x(0)=d-B \int_{0}^{\tau} y(s) d s \\
& -B \sum_{0<t_{j}<\tau} a_{j}-C \int_{0}^{T} y(s) d s-C \sum_{0<t_{j}<T} a_{j} .
\end{aligned}
$$

Thus, we obtain

$$
\begin{aligned}
& x(0)=N^{-1} d-N^{-1} B \int_{0}^{\tau} y(s) d s-N^{-1} B \sum_{0<t_{j}<\tau} a_{j} \\
& -N^{-1} C \int_{0}^{T} y(s) d s-N^{-1} C \sum_{0<t_{j}<T} a_{j} .
\end{aligned}
$$


So putting (9) in (7), we get

$$
\begin{aligned}
& x(t)=N^{-1} d-N^{-1} B \int_{0}^{\tau} y(s) d s-N^{-1} B \sum_{0<t_{j}<\tau} a_{j} \\
& -N^{-1} C \int_{0}^{T} y(s) d s-N^{-1} C \sum_{0<t_{j}<T} a_{j}+\int_{0}^{t} y(s) d s+\sum_{0<t_{j}<t} a_{j} .
\end{aligned}
$$

Now consider $t \in\left(t_{j}, t_{j+1}\right], t_{j+1}<\tau$. Then we can rewrite equality (10) as follows:

$$
\begin{aligned}
x(t)= & N^{-1} d-N^{-1} B \int_{0}^{t} y(s) d s-N^{-1} B \int_{t}^{\tau} y(s) d s-N^{-1} B \sum_{0<t_{j}<t} a_{j} \\
& -N^{-1} B \sum_{t<t_{j}<\tau} a_{j}-N^{-1} C \int_{0}^{t} y(s) d s-N^{-1} C \int_{t}^{\tau} y(s) d s \\
& -N^{-1} C \int_{\tau}^{T} y(s) d s-N^{-1} C \sum_{0<t_{j}<t} a_{j}-N^{-1} C \sum_{t<t_{j}<\tau} a_{j} \\
- & N^{-1} C \sum_{\tau<t_{j}<T}^{t} a_{j}+\int_{0} y(s) d s+\sum_{0<t_{j}<T} a_{j} .
\end{aligned}
$$

Grouping the terms and then simplifying, we get

$$
\begin{aligned}
& x(t)=N^{-1} d+N^{-1} A \int_{0}^{t} y(s) d s-N^{-1}(B+C) \int_{t}^{\tau} y(s) d s \\
& -N^{-1} C \int_{\tau}^{T} y(s) d s+N^{-1} A \sum_{0<t_{j}<t} a_{j} \\
& -N^{-1}(B+C) \sum_{t<t_{j}<\tau} a_{j}-N^{-1} C \sum_{\tau<t_{j}<T} a_{j} .
\end{aligned}
$$

Let us define the new function as follows:

$$
G_{1}(t, s)=\left\{\begin{array}{lr}
N^{-1} A, & 0 \leq s \leq t, \\
-N^{-1}(B+C), & t \leq s \leq \tau, \\
-N^{-1} C, & \tau<s \leq T .
\end{array}\right.
$$


Using this function in (10), we obtain the following result

$$
x(t)=D+\int_{0}^{T} G_{1}(t, s) y(\tau) d \tau+\sum_{0<t_{k}<T} G_{1}\left(t_{j}, t_{k}\right) a_{k} .
$$

For the case $t \in\left(t_{j}, t_{j+1}\right], t_{j}>\tau$ we can write equality (10) as follows

$$
\begin{gathered}
x(t)=N^{-1} d-N^{-1} B \int_{0}^{\tau} y(s) d s-N^{-1} B \sum_{0<t_{j}<\tau} a_{j} \\
-N^{-1} C \int_{0}^{\tau} y(s) d s-N^{-1} C \int_{\tau}^{t} y(s) d s-N^{-1} C \int_{t}^{T} y(s) d s \\
-N^{-1} C \sum_{0<t_{j}<\tau} a_{j}-N^{-1} C \sum_{\tau<t_{j}<t} a_{j}-N^{-1} C \sum_{t<t_{j}<T} a_{j}+\int_{0}^{\tau} y(s) d s \\
+\int_{\tau}^{t} y(s) d s+\sum_{0<t_{j}<\tau} a_{j}+\sum_{\tau<t_{j}<t} a_{j}=N^{-1} d+N^{-1} A \int_{0}^{\tau} y(s) d s \\
+N^{-1}(A+B) \int_{\tau}^{t} y(s) d s-N^{-1} C \int_{t}^{T} y(s) d s+N^{-1} A \sum_{0<t_{j}<\tau} a_{j} \\
+N^{-1}(A+B) \sum_{\tau<t_{j}<t} a_{j}-N^{-1} C \sum_{t<t_{j}<T} a_{j} .
\end{gathered}
$$

Here we introduce the new function

$$
G_{2}(t, s)=\left\{\begin{array}{lc}
N^{-1} A, & 0 \leq s \leq \tau, \\
N^{-1}(A+B), & \tau<s \leq t \\
-N^{-1} C, & t<s \leq T .
\end{array},\right.
$$

Thus, for each $t \in\left(t_{j}, t_{j+1}\right]$, we have

$$
x(t)=N^{-1} d+\int_{0}^{T} G_{2}(t, \tau) y(\tau) d \tau+\sum_{0<t_{k}<T} G_{2}\left(t_{j}, t_{k}\right) a_{k} .
$$


Consequently, we deduce that the solution of boundary value problem (4)-(6) is in the form

$$
x(t)=D+\int_{0}^{T} G(t, s) y(s) d s+\sum_{0<t_{k}<T} G\left(t_{j}, t_{k}\right) a_{k} .
$$

The proof is completed.

Remark 3.3. Note that for solution (7) we have

(i) $D=N^{-1} d$ is the solution of $\dot{x}(t)=0$ with nonlocal boundary condition $A x(0)+B x(\tau)+C x(T)=d ;$

(ii) The function $\int_{0}^{T} G(t, \tau) y(\tau) d \tau$ is the solution of $\dot{x}(t)=y(t)$ with nonlocal boundary condition $A x(0)+B x(\tau)+C x(T)=0$. Here $G(t, \tau)$ is Green's function of problem (4)-(6);

(iii) The functions $\sum_{0<t_{k}<T} G\left(t_{i}, t_{k}\right) a_{j}, i=1,2, \ldots, p$ are the solutions of $\dot{x}(t)=0$ with nonlocal boundary condition $A x(0)+B x(\tau))+C x(T)=0$ and jumps (5).

Lemma 3.4. Assume that $f \in C\left([0, T] \times R^{n}, R^{n}\right)$ and $I_{k}(x) \in C\left(R^{n}\right), k=$ $1,2, \ldots, p$. Then the function $x(t)$ is a solution of impulsive boundary value problem (1)-(3) if and only if $x(t)$ is a solution of the impulsive integral equation

$$
\begin{aligned}
& x(t)=D+\int_{0}^{T} G(t, s) f(s, x(s)) d s+\sum_{k=1}^{p} G\left(t_{i}, t_{k}\right) I_{k}\left(x\left(t_{k}\right)\right) \\
& \text { for } t \in\left(t_{i}, t_{i+1}\right], i=0,1, \ldots, p .
\end{aligned}
$$

Proof. Let $x(t)$ be a solution of boundary-value problem (1)-(3). Analogously, as in Lemma 3.2 we can prove that the impulsive integral equation (13) is also a solution of boundary value problem (1)-(3). Clearly, the solution of impulsive integral equation (13) satisfies the boundary value problem (1)-(3). Lemma 3.4 is proved.

\section{Main results}

Now we present the existence uniqueness result for problem (1)-(3). Our considerations are based on the Banach fixed point theorem. 
Theorem 4.1. Assume that:

(H1) There exists a constant $M>0$ such that

$$
|f(t, x)-f(t, y)| \leq M|x-y|
$$

for any $t \in[0, T]$ and all $x, y \in R^{n}$.

(H2) There exist constants $l_{i}>0, i=1,2, \ldots, p$ such that

$$
\left|I_{i}(x)-I_{i}(y)\right| \leq l_{i}|x-y|
$$

for all $x, y \in R^{n}$

If

$$
L=S\left(M T+\sum_{k=1}^{p} l_{k}\right)<1,
$$

then the boundary value problem (1)-(3) has a unique solution on $[0, T]$. Here

$$
S=\max _{[0, T] \times[0, T]}\|G(t, s)\| .
$$

Proof. We transform the problem (1)-(3) into a fixed point problem. Let the operator

$$
F: P C\left([0, T], R^{n}\right) \rightarrow P C\left([0, T], R^{n}\right),
$$

be defined as

$$
F(x)(t)=\int_{0}^{T} G(t, s) f(s, x(s)) d s+\sum_{k=1}^{p} G\left(t_{i}, t_{k}\right) I_{k}\left(x\left(t_{k}\right)\right)
$$

for $t \in\left(t_{i}, t_{i+1}\right], i=0,1, \ldots, p$. We will show that $F$ is a contraction. Consider $x, y \in P C\left([0, T], R^{n}\right)$. Then for each $t \in\left(t_{i}, t_{i+1}\right]$,

$$
\begin{aligned}
\mid F(x)(t)- & F(y)(t)\left|\leq \int_{0}^{T}\right| G(t, s)|| f((s, x(s))-f((s, y(s)) \mid d s \\
& +\sum_{k=1}^{p} \mid G\left(t_{i}, t_{k}|| I_{k}\left(x\left(t_{k}\right)\right)-I_{k}\left(y\left(t_{k}\right)\right) \mid\right. \\
\leq & S M T\|x-y\|+S \sum_{k=1}^{p} l_{k}\left|x\left(t_{k}\right)-y\left(t_{k}\right)\right|
\end{aligned}
$$




$$
\leq\left[S\left(M T+\sum_{k=1}^{p} l_{k}\right)\right]\|x-y\|_{P C} .
$$

Then

$$
\|F(x)(t)-F(y)(t)\|_{P C} \leq L\|x-y\|_{P C},
$$

showing that $F$ is a contraction, and thus it has a unique fixed point. So, the operator $F$ is a solution of problem (1)-(3).

Finally, the existence and uniqueness result for the solutions of the problem (1)-(3) make use of the Banach fixed point principle. Theorem 4.1 is proved.

Now we present the existence result for the problem (1)-(3) that is based on the Schaefer's fixed point theorem.

Theorem 4.2. Assume that:

(H3) The function $f:[0, T] \times R^{n} \rightarrow R^{n}$ is continuous.

(H4) There exists a constant $M_{1}>0$ such that $|f(t, x)| \leq M_{1}$ for any $t \in[0, T]$ and all $x \in R^{n}$.

(H5) The functions $I_{k}(x), x \in R^{n}, k=1,2, \ldots, p$ are continuous and there exists a constant $M_{2}>0$ such that

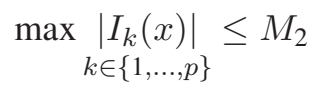

for all $x \in R^{n}$.

Then boundary value problem (1)-(3) has at least one solution on [0,T].

Proof. We will show that under the assumptions of the theorem, the operator $F$ has a fixed point. The proof will be given in several steps.

Step 1. Here we prove that $F$ is continuous.

Let $\left\{x_{n}\right\}$ be a sequence such that $x_{n} \rightarrow x$ in $P C\left([0, T], R^{n}\right)$. Then for any

$$
\begin{gathered}
t \in\left(t_{i}, t_{i+1}\right], i=0,1, \ldots, p, \\
\left|F\left(x_{n}\right)(t)-F(x)(t)\right| \\
\leq \int_{0}^{T}|G(t, s)|\left|f\left(s, x_{n}(s)\right)-f(s, x(s))\right| d s \\
+\sum_{k=1}^{p} \mid G\left(t_{i}, t_{k}|| I_{k}\left(x_{n}\left(t_{k}\right)\right)-I_{k}\left(x\left(t_{k}\right)\right) \mid\right. \\
\leq S T \max _{s \in[0, T]}\left|f\left(s, x_{n}(s)\right)-f(s, x(s))\right|
\end{gathered}
$$




$$
+S \sum_{k=1}^{p}\left|I_{k}\left(x_{n}\left(t_{k}\right)\right)-I_{k}\left(x\left(t_{k}\right)\right)\right| .
$$

Since $f$ and $I_{k}, k=1,2, \ldots, p$ are continuous functions, we have

$$
\left\|F\left(x_{n}\right)(t)-F(x)(t)\right\|_{P C} \rightarrow 0
$$

as $n \rightarrow \propto$. So, $F$ is continuous.

Step 2. In this step, we prove that the operator $F$ maps bounded sets in $P C\left([0, T], R^{n}\right)$.

It is enough to show for any $\eta>0$, there exists a positive constant $l$ such that for any $\left\{x \in B_{\eta}=P C\left([0, T], R^{n}\right):\|x\| \leq \eta\right\}$, we have $\|F(x(\cdot))\| \leq l$. Applying triangle inequality, assumptions (H4) and (H5), for $t \in\left(t_{i}, t_{i+1}\right]$ we get

$$
|F(x)(t)| \leq|D|+\int_{0}^{T}|G(t, s)||f(s, x(s))| d s+\sum_{k=1}^{p}\left|G\left(t_{i}, \tau_{k}\right)\right|\left|I_{k}\left(t_{k}\right)\right|
$$

for any $t \in[0, T]$. Hence,

$$
|F(x)(t)| \leq|D|+S T M_{1}+S p M_{2} .
$$

So,

$$
\|F(x)(t)\|_{P C} \leq|D|+S\left[T M_{1}+p M_{2}\right]=l .
$$

Step 3. The purpose of this step is to prove that the operator $F$ maps bounded sets into equicontinuous sets of $P C\left([0, T], R^{n}\right)$.

Let $\xi_{1}, \xi_{2} \in[0, T], \xi_{1}<\zeta_{2}, B_{\eta}$ be a bounded set of $P C\left([0, T], R^{n}\right)$ as in Step 2 , and assume that $x \in B_{\eta}$.

Case 1: $\xi_{1}, \xi_{2} \in\left[t_{j}, t_{j+1}\right], t_{j+1}<\tau$. Then,

$$
\begin{aligned}
& \left|F(x)\left(\xi_{2}\right)-F(x)\left(\xi_{1}\right)\right| \\
= & \left|N^{-1} A \int_{\zeta_{1}}^{\xi_{2}} f(s, x(s)) d s+N^{-1}(B+C) \int_{\xi_{1}}^{\xi_{2}} f(s, x(s)) d s\right| \\
\leq & \int_{\xi_{1}}^{\xi_{2}}|f(s, x(s))| d s .
\end{aligned}
$$


Case 2: Let $\xi_{1} \in\left[t_{p_{1}}, \tau\right], \xi_{2} \in\left[\tau, t_{p_{1}+1}\right]$. Then,

$$
\begin{aligned}
\left|F(x)\left(\xi_{2}\right)-F(x)\left(\xi_{1}\right)\right| & =\left|\int_{\xi_{1}}^{\tau} f(s, x(s)) d s+\int_{\tau}^{\xi_{2}} f(s, x(s)) d s\right| \\
& \leq \int_{\xi_{1}}^{\xi_{2}}|f(s, x(s))| d s .
\end{aligned}
$$

Case 3: $\xi_{1}, \xi_{2} \in\left[t_{j}, t_{j+1}\right], t_{j}>\tau$.

We prove Case 3 in the similar way as the Case 1. So,

$$
\left|F(x)\left(\xi_{2}\right)-F(x)\left(\xi_{1}\right)\right| \leq \int_{\xi_{1}}^{\xi_{2}}|f(s, x(s))| d s .
$$

The right-hand side of the above inequalities for all three cases tends to zero as $\xi_{1} \rightarrow \xi_{2}$. As a consequence of Steps 1,2 and 3 together with the Arzela-Ascoli theorem, we can conclude that the operator $F: P C\left([0, T], R^{n}\right) \rightarrow P C\left([0, T], R^{n}\right)$ is completely continuous.

Step 4. Now it remains to show that the set

$$
\Delta=\left\{x \in P C\left([0, T], R^{n}\right): x=\lambda F(x) \text { for some } 0<\lambda<1\right\}
$$

is bounded. Assume that $x=\lambda(F x)$ for some $0<\lambda<1$. Thus, for any $t \in\left(t_{i}, t_{i+1}\right], i=0,1, \ldots, p$ we have

$$
x(t)=\lambda\left[D+\int_{0}^{T} G(t, s) f(s, x(s)) d s+\sum_{k=1}^{p} G\left(t_{i}, \tau_{k}\right) I_{k}\left(x\left(t_{k}\right)\right)\right] .
$$

This fact in combination with (H4) and (H5) shows that for any $t \in[0, T]$ we have

$$
|F(x)(t)| \leq|D|+\left[M_{1} T+p M_{2}\right] S .
$$

So,

$$
\|x\|_{P C} \leq|D|+\left[M_{1} T+p M_{2}\right] S=R .
$$

This shows that the set $\Delta$ is bounded and $F$ has a fixed point by Schaefer's fixed point theorem, that is a solution of (1)-(3). Theorem 4.2 is proved. 


\section{Examples}

Example 5.1. Let us consider the following there-point boundary value problem for a system of impulsive differential equations with three-point boundary conditions:

$$
\left\{\begin{array}{l}
\dot{x}_{1}=0.2 \cos x_{2}(t), \quad t \in(0,2), t \neq \frac{1}{2}, \\
\dot{x}_{2}=0.2 \sin x_{1}(t), \quad t \in(0,2), t \neq \frac{1}{2}
\end{array}\right.
$$

with

$$
\left\{\begin{array}{l}
x_{1}(0)-\frac{1}{2} x_{2}(0)+x_{2}(1)-\frac{1}{2} x_{2}(2)=1 \\
-\frac{1}{2} x_{1}(0)+x_{1}(1)-\frac{1}{2} x_{1}(2)+x_{2}(2)=1
\end{array}\right.
$$

and

$$
\Delta x_{1}\left(\frac{1}{2}\right)=\frac{1}{10} x_{2}\left(\frac{1}{2}\right), \Delta x_{2}\left(\frac{1}{2}\right)=\frac{1}{10} x_{1}\left(\frac{1}{2}\right) .
$$

It is clear that

$$
\begin{gathered}
A=\left(\begin{array}{cc}
1 & -\frac{1}{2} \\
-\frac{1}{2} & 0
\end{array}\right), \quad B=\left(\begin{array}{ll}
0 & 1 \\
1 & 0
\end{array}\right), C=\left(\begin{array}{cc}
0 & -\frac{1}{2} \\
-\frac{1}{2} & 1
\end{array}\right), \\
d=\left(\begin{array}{l}
1 \\
1
\end{array}\right), A+B+C=\left(\begin{array}{ll}
1 & 0 \\
0 & 1
\end{array}\right) .
\end{gathered}
$$

Here $S \leq 1,5, M=0.2, l=0.1$. Thus, the conditions (H1) and (H2) hold with $M=0.2, l_{1}=0.1$. We can easily see that condition (14) is satisfied:

$$
L=S\left(M T+\sum_{i=1}^{p} l_{i}\right)=1.5(0.2 \cdot 2+0.1)=0.75<1 .
$$

By Theorem 4.1, the boundary value problem (16)-(18) has a unique solution on $[0,2]$.

Example 5.2. Consider the following boundary value problem

$$
\begin{cases}\dot{x_{1}}=\frac{1}{1+\sin ^{2} x_{2}}, & t \in(0,2), \\ \dot{x_{2}}=\frac{1}{1+\cos ^{2} x_{1}}, & t \in(0,2),\end{cases}
$$

subject to impulsive conditions

$$
\left\{\begin{aligned}
\Delta x_{1}(0.25) & =\frac{1}{1+x_{2}^{2}(0.25)}, \\
\Delta x_{2}(1.5) & =\frac{1}{1+x_{2}^{2}(1.5)},
\end{aligned}\right.
$$


and three-point boundary conditions

$$
\begin{aligned}
& x_{1}(0)+x_{2}(1)-x_{1}(2)=-1 \\
& x_{2}(0)+x_{1}(1)-2 x_{2}(2)=1 .
\end{aligned}
$$

Obviously,

$$
\begin{gathered}
A=\left(\begin{array}{ll}
1 & 0 \\
0 & 1
\end{array}\right), B=\left(\begin{array}{ll}
0 & 1 \\
1 & 0
\end{array}\right), C=\left(\begin{array}{cc}
-1 & 0 \\
0 & -2
\end{array}\right), d=\left(\begin{array}{c}
-1 \\
1
\end{array}\right) \\
N=A+B+C=\left(\begin{array}{cc}
0 & 1 \\
1 & -1
\end{array}\right)
\end{gathered}
$$

and the functions, $\left(\begin{array}{l}f_{1} \\ f_{2}\end{array}\right)=\left(\begin{array}{c}\frac{1}{1+\sin ^{2} x_{2}} \\ \frac{1}{1+\cos ^{2} x_{1}}\end{array}\right),\left(\begin{array}{c}I_{1} \\ I_{2}\end{array}\right)=\left(\begin{array}{c}\frac{1}{1+x_{2}^{2}} \\ \frac{1}{1+x_{1}^{2}}\end{array}\right)$ are continuous and bounded. Hence, conditions (H3)-(H5) hold. By Theorem 4.2, the boundary-value problem (20)-(22) has at least one solution on $[0,2]$.

\section{Conclusion}

In this research, the existence and uniqueness of solutions for nonlinear impulsive differential equations with three-point boundary conditions have been studied by using some well-known fixed point theorems. The technique used in this research can be applied to the similar problems for impulsive differential equations subject to multi-point nonlocal conditions

$$
E x(0)+\sum_{j=1}^{J} B_{j} x\left(\lambda_{j}\right)=d
$$

and impulsive conditions

$$
x\left(\tau_{i}^{+}\right)-x\left(\tau_{i}\right)=a_{i}, i=1,2, \ldots, p, t \in[0, T], 0=\tau_{0}<\tau_{1}<\ldots<\tau_{p}<T,
$$

where $E$ is a unit matrix, $B_{j} \in R^{n \times n}$ are given matrices and

$$
\sum_{j=1}^{J}\left\|B_{j}\right\|<1, \quad 0<\lambda_{1}<\ldots<\lambda_{j} \leq T
$$




\section{Bibliography}

[1] M. Benchohra, J. Henderson and J. Ntouyas, Impulsive Differential Equations and Inclusion, Vol. 2, Hindawi Publishing, New York, 2006.

[2] D. D. Bainov and P. S. Simeonov, Systems with Impulsive Effect, Horwood, Chichister, 1989.

[3] V. Lakshmikantham, D. D. Bainov. and P. S. Simeonov, Theory of Impulsive Differential Equations, World Scientific, Singapore, 1989.

[4] A. M. Samoilenko and N. A. Perestyuk, Impulsive Differential Equations, World Scientific, Singapore, 1995.

[5] S. Timoshenko, Theory of Elastic Stability, McGraw-Hill, New-York, 1961.

[6] A. Biglan, D. Ary and A. C. Wagenaar, The value of interrupted time-series experiments for community intervention research, Prevention Science: The official Journal of the Society for Prevention Research 1(1) (2000), 31-49.

[7] C. Day, L. Degenhardt, S. Gilmour and W. Hall, Effects of supply reduction upon injecting drug use, British Medical Journal 329 (2004), 428-429.

[8] A. Dishliev, K. Dishlieva and S. Nenov, Specific Asymptotic Properties of the Solutions of Impulsive Differential Equations: Methods and Applications, Academic Publications, 2012.

[9] J. M. Gallardo, Second order differential operators with integral boundary conditions and generation of semigroups, Rocky Mountain J. Math. 30 (2000), 1265-1291.

[10] M. Benchohra, F. Berhoun and J. Henderson, Nonlinear impulsive differential inclusions with integral boundary conditions, Commun. Math. Anal. 5(2) (2008), 60-75.

[11] M. Feng, B. Du and W. Ge, Impulsive boundary value problems with integral boundary conditions and one-dimensional p-Laplacian, Nonlinear Anal. 70 (2009), 31193126.

[12] J. J. Nieto and R. Rodriguez-Lopez, New comparision results for impulsive integrodifferential equations and applications, J. Math. Anal. Appl. 328 (2007), 1343-1368.

[13] J. J. Nieto and R. Rodriguez-Lopez, Boundary value problems for a class of impulsive functional equations, Comput. Math. Appl. 55 (2008), 2715-2731.

[14] M. Benchohra, F. Berhoun and J. Henderson, Multiple positive solutions for impulsive boundary value problem with integral boundary conditions, Math. Sci. Res. J. 11(12) (2007), 614-626.

[15] J. J. Nieto and C. C. Tisdell, Existence and uniqueness of solutions to first-order systems of nonlinear impulsive boundary value problems with sub-, super-linear or linear growth, Electron. J. Differ. Equ. 2007(105) (2007), 1-14.

[16] M. J. Mardanov and Y. A. Sharifov, Existence results for first order nonlinear impulsive differential equations with nonlocal boundary conditions, AIP Conference Proceedings 1676 (2015), 020015, doi: 10.1063/1.4930441. 
[17] A. Ashyralyev and Y. A. Sharifov, Existence and uniqueness of solutions for nonlinear impulsive differential equations with two-point and integral boundary conditions, Advances in Difference Equations 2013(173) (2013), 1-11, doi: 10.1186/1687-18472013-173.

[18] A. Ashyralyev and Y. A. Sharifov, Optimal control problems for impulsive systems with integral boundary conditions, Electronic Journal of Differential Equations 2013(80) (2013), 1-11.

[19] Y. A. Sharifov, Optimal control of impulsive systems with nonlocal boundary conditions, Russian Math. 57(2) (2013), 65-72.

[20] V. M. Abdullayev, Numerical solution to optimal control problems with multipoint and integral conditions, Proc. Inst.Math. Mech. Natl. Acad. Sci Azerb. 44(2) (2018), 171-186.

[21] K. R. Aida-zade, An approach for solving nonlinear loaded problems for linear ordinary differential equations, Proc. Inst.Math. Mech. Natl. Acad. Sci Azerb. 44(2) (2018), 338-350.

[22] R. A. Khan, Existence and approximation of solutions of nonlinear problems with integral boundary conditions, Dyn. Syst. Appl. 14 (2005), 281-296.

[23] M. Benchohra, S. Hamani and J. J. Nieto, The method of upper and lower solutions for second order differential inclusions with integral boundary conditions, Rocky Mt. J. Math. 40(1) (2010), 13-26.

[24] R. Y. Ma, Existence theorems for a second order three-point boundary value problem, J. Math. Anal. Appl 212 (1997), 430-442.

[25] Y. A. Sharifov and K. E. Ismayilova, Existence and uniqueness of solutions to threepoint boundary value problems, Proceedings of the 6th International Conference on Control and Optimization with Industrial Applications, Vol.II, 11-13 July, 2018 , Baku, Azerbaijan, 271-273.

[26] A. Boucherif, Second order boundary value problems with integral boundary condition, Nonlinear Anal. 70 (2009), 364-371.

[27] M. Denche and A. Kourta, Boundary value problem for second order differential operators with integral boundary conditions, Appl. Anal. 84 (2005), 1247-1266.

[28] N. Sveikate, On three-point boundary value problem, Mathematical Modelling and Analysis 21(2) (2016), 270-281.

[29] T. Jankowski, Differential equations with integral boundary conditions, J. Comput. Appl. Math. 147 (2002), 1-8.

Received December 26, 2018; revised February 15, 2019; accepted March 22, 2019. 


\section{Author information}

Misır J. Mardanov, Institute of Mathematics and Mechanics of NAS of Azerbaijan, Azerbaijan.

E-mail: misirmardanov@yahoo.com

Yagub A. Sharifov, Institute of Mathematics and Mechanics of NAS of Azerbaijan \& Baku State University, Azerbaijan.

E-mail: sharifov22@rambler.ru

Kamala E. Ismayilova, Baku Engineering University, Khirdalan city, 120 AZ0102, Absheron, Baku, Azerbaijan.

E-mail: keismayilova@beu.edu.az 